\title{
Effect of Total Proteoglycans on Cell Proliferation and Tumor Formation in Mice
}

\author{
Valentina I. Rykova ${ }^{1}$, Galina M. Ronichevskaya ${ }^{1}$ and Elvira V. Grigorieva ${ }^{2}$ \\ ${ }^{1}$ Department of Genome Structure, Institute of Cytology and Genetics SD RAS, Novosibirsk, \\ 630090, Russia; ${ }^{2}$ Laboratory of Molecular Mechanisms of Carcinogenesis, Institute of \\ Molecular Biology and Biophysics SD RAMS, Novosibirsk, 630117, Russia
}

KEYWORDS Proteoglycan. Tumor. Heparan Sulfate. Dermatan Sulfate. Cell Proliferation

\begin{abstract}
Proteoglycans (PGs) are heterogeneous macromolecules that can both suppress and induce mitotic activity and cell growth depending on their structure and experimental conditions. Possibly, their combination is necessary for the proper regulatory effect to the certain tissue. In the present study, an influence of bovine liver total proteoglycans on the mitotic activity of different mouse embryonic tissues and mouse adenocarcinoma cells was investigated. Bovine liver proteoglycans suppressed cell proliferation in the mouse liver $(1.8 \%$ mitotic cells versus $10.6 \%$ in the control animals) but not in the other tissues. The antimitotic effect of the PGs was tissue-specific as it was shown by experiments with bovine spleen and lung PG preparations. An influence of the total proteoglycan preparation on tumor cell proliferation was studied by treatment of primary breast adenocarcinoma cells with bovine liver PGs before injection into experimental animals. The treatment suppressed tumor formation in mice - only $20 \%$ animals developed tumors on $26^{\text {th }}$ day and $55 \%$ ones on $43^{\text {rd }}$ day (versus $75 \%$ and $100 \%$ in a control group, respectively). Intraperitoneal injection of the proteoglycans into mice with induced Ehrlich ascite carcinoma decreased tumor weight and tumor volume by $50 \%$. These results indicate that bovine liver total proteoglycans are able to suppress cell proliferation in both fetal mouse tissues and orthotopic mammary carcinoma model in vivo. The effect is tissue-specific and species non-specific with optimal concentration $0.3-0.4 \mathrm{mg} / \mathrm{g}$ animal weight. The data suggest that total bovine proteoglycan preparations may be potential antimitotic compound to cell proliferation disorders for another animal species.
\end{abstract}

\section{INTRODUCTION}

Proteoglycans (PGs) are complex macromolecules consisted of core protein to which glycosaminoglycan (GAGs) chains are attached. Cell surface proteoglycans are known to mediate many aspects of cell behavior including cell adhesion, modulation of growth factor activity, cell migration and proliferation (Kresse and Schonherr 2001). Modification of cell surface proteoglycans and their composition is one of the critical steps involved in cellular transformation and is thought to play key regulatory functions during various stages of tumor development (Selleck 2000; Iozzo 1998). Among the different proteoglycan molecules, some emerged as inhibitors of cell proliferation while another as stimulators of the process (Timar et al. 2002). It was shown that decorin, a small leucine-rich dermatan sulfate proteoglycan, is able to suppress the growth of various tumor

Correspondence to: Dr. E.Grigorieva,

Laboratory of Molecular Mechanisms of Carcinogenesis, Institute of Molecular Biology and Biophysics SD RAMS,

Timakova 2, 630117, Russia

E-mail: elv_grig@yahoo.com cell lines of various histogenetic backgrounds through the EGF receptor/MAP kinase/ p21 axis (Moscatello et al. 1998; Santra et al. 2000). Keratansulfate proteoglycan lumican also is involved in the control of cell proliferation and may be considered, like decorin, as an anti-tumor factor from the extracellular matrix (Vuillermoz et al. 2004; Vij et al. 2004). For some proteoglycans an inverse or ambiguous effects have been shown. Heparansulfate proteoglycan syndecan1 is involved in the promotion of the cell proliferation (Maeda et al. 2004; Barbareschi et al. 2003); glypican-3 can both inhibit cell proliferation and induce it depending on the tissue (Filmus 2001). Perlecan may inhibit the growth and invasiveness of fibrosarcoma cells (Mathiak et al. 1997) and serum-stimulated SMC growth (Garl et al. 2004), but it induces tumor growth and angiogenesis in vivo (Sharma et al. 1998).

Controversial results were obtained also in experiments on common effects of GAGs of the same class on cell culture in vitro. Different glycosaminoglycans were tested to evaluate their effects on proliferation of various cell lines. It was shown that heparin $(\mathrm{He})$ is able to inhibit cell proliferation, while chondroitin sulfates (CS) and dermatan sulfates (DS) significantly stimulate 
cell growth of rhabdomyosarcoma (RMS) (Redini et al. 1990) and monoblastic leukemia cell line (U937) (Volpi et al. 1994). However for osteoblastic cell lines, an inhibitory effect of CS and DS was shown (Nikitovic et al. 2005). In general, the regulator influence of different GAG kind was inconsistent and some GAGs developed opposite effect depending on the fast or slow growth conditions (Nagasawa et al. 1993) and presence of serum in the cultural medium (Maurer et al. 1994). It is known that every tissue contains a complex combination of different proteoglycans which ratio may vary with the tissue state. Taking into account inverse capabilities of different proteoglycans to influence cell proliferation, in many cases it is hard to predict their integral effect on mitotic activity of the cells. This study is the first to show a combined influence of total proteoglycan preparation from different bovine tissues on cell proliferation and tumor growth in vivo.

\section{MATERIALS AND METHODS}

Animals and Tissues: Adult (2-month-old) female mice $\mathrm{C} 3 \mathrm{H}$ and 5-9 days old mice $\mathrm{C} 57 \mathrm{Bl}$ were kept in the special facilities (Animal Cure Facility of Institute of Cytology and Genetics, Novosibirsk, Russia) and maintained on a standard diet and tap water ad libitum. The experiment protocol was approved by the local ethics committee for animal studies. All bovine tissues were collected at a local abattoir (Novosibirsk, Russia), within 20 min of slaughter and immediately frozen in liquid nitrogen.

Isolation of Total Proteoglycans: Total proteoglycan preparations were isolated from different bovine tissues by phenol deproteinization according to (Kirby 1956) with some additional steps. Briefly, tissue was homogenized with $0.14 \mathrm{M} \mathrm{NaCl} / 0.01 \mathrm{M}$ Trilon B solution (1:9) and deproteinized by shaking with an equal volume of $90 \%$ phenol (5000rpm, $40 \mathrm{~min}$ ) twice. Proteoglycans were precipitated from water phase by addition of 2 volumes of cold absolute ethanol and $\mathrm{NaAc}$ up to $2 \%$ followed by overnight incubation at $20{ }^{\circ} \mathrm{C}$. Precipitated material was collected by centrifugation at $4{ }^{\circ} \mathrm{C}$ $(5,000 \mathrm{rpm}$ for $10 \mathrm{~min})$, dissolved in water and ultracentrifuged at $110000 \mathrm{~g}$ for $2 \mathrm{~h}$. Then, $\mathrm{HClO} 4$ was slowly added to supernatant up to $0,5 \mathrm{M}$ final concentration (on ice) and acid-insoluble material was removed by centrifugation at 5000rpm for 10min. Supernatant was neutralized with $2 \mathrm{M}$ $\mathrm{NaOH}$ (up to $\mathrm{pH} 5-6$ ) and proteoglycans were precipitated as described above. Each pellet was dissolved in water and re-pelleted again. The final pellet was reconstituted in distilled water and applied to a DEAE-Sephadex 25 column. The column was rinsed with water and then eluted sequentially with $0,4 \mathrm{M} \mathrm{NaAc} / 1 \%$ Triton $\mathrm{X}-100 /$ $8 \mathrm{M}$ urea and 2,2M NaAc/1\% Triton $\mathrm{x}-100 / 8 \mathrm{M}$ urea. Proteoglycans were precipitated from the last fraction with ethanol/NaAc twice as described above. The last pellet was rinsed with ethanol and lyophilized to dryness.

Glycosaminoglycan Characterization: Proteoglycan composition of the total preparation was identified by chondroitinase digestion and nitrous acid treatment. For chondroitinsulfate/ dermatansulfate identification, aliquots were treated with chondroitinase ACII lyase (EC 4.2.2.5; Sigma) or with chondroitinase ABC lyase (EC 4.2.2.4; Sigma) according the manufacturer's instructions. Heparansulfates were identified by nitrous acid digestion: equal parts of $1 \mathrm{M} \mathrm{H} 2 \mathrm{SO} 4$ were mixed with $0.5 \mathrm{M}$ barium nitrite hydrate at room temperature, and the precipitate was separated out by centrifugation at 10,000 rpm (Eppendorf 5415c) for $5 \mathrm{~min}$ at room temperature. Lyophilized extract samples were resuspended in the supernatant (nitrous acid, $\mathrm{pH} 1.5$ ), incubated for $20 \mathrm{~min}$ at room temperature, and then neutralized with a volume of $1 \mathrm{MNaHCO}$.

Cells Proliferation Assay in Mouse Tissues in Vivo: Lyophilized proteoglycans preparation was diluted with the saline and injected subcutaneously to 5-9 days old mice C57Bl. Control animals got the equal amount of saline. Totally, 20 mice were used for the experiments with liver proteoglycan preparation, 10 mice for spleen and 10 mice for lung proteoglycan preparations. Animals were sacrificed by decapitation after 1,3,5,7 and 19 hours. Tissues were fixed with Carnua reagent and mitotic activity of the cells was counted on acetocarmin stained samples as percentage of dividing cells. 5-20*103 cells have been checked for every tissue sample.

Influence of Proteoglycans on Primary Cell Culture: Primary cell culture was done from spontaneous mouse breast adenocarcinoma, developed in $\mathrm{C} 3 \mathrm{H}$ female mice. Cells were cultured in medium 199 (40\%), lactalbumin hydrolyzed (40\%) and 20\% FBS. Medium was changed after 24 hours of seeding of the cells and PG preparation was added to concentrations 
0,003; 0,006; $0,012 \ldots 24 \mathrm{mg} / \mathrm{ml}$. Control cultures was treated with the appropriate amount of saline. After next 24 hours slides were fixed with a mixture acetic asid:methanol (1:3), stained with gaematoxilin-eosin and embedded into the Canadian Balm. Cell proliferation intensity was estimated by counting of dividing cells in series $1-2,5 * 104$ cells.

Influence of Proteoglycans on Growth of Tumor Cells Inoculated in vivo: Mouse adenocarcinoma cells from primary cell culture were treated with total PG preparation $(6 \mathrm{mg} / \mathrm{ml}$ final concentration) for $10 \mathrm{~min}$. Then cells were injected subcutaneously to the female mice $\mathrm{C} 3 \mathrm{H}$ as $1,2 * 105$ cells/mouse. Untreated tumor cells were injected as a control. Each experiment was done in series of 20 animals. Rate of tumor lesions formation and a tumor size were counted in each series.

\section{RESULTS}

Proteoglycan Composition in the Different Bovine Tissues: Proteoglycans were extracted from bovine liver, kidney, lung and breast tissues as described in Methods. GAG composition was identified by appropriate enzymatic digestion with chondroitinase ACII lyase to digest CS side chains or with chondroitinase $\mathrm{ABC}$ lyase to digest both CS and DS side chains or by nitrous acid treatment with consequent agarose gel electrophoresis. Amount of every GAG type was determined as relative percentages by densitometric scanning of Alcian Blue stained strips (Fig.1).

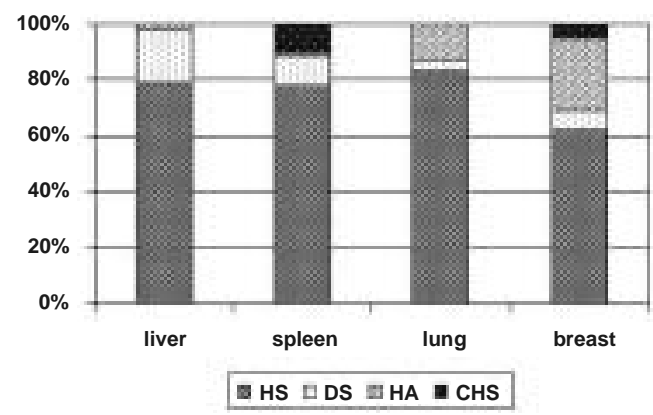

Fig. 1. The composition of proteoglycan preparations isolated from different bovine tissues. PGs were separated by electrophoresis in agarose gel, stained with Alcian Blue and quantified by densitometry. HS, heparan sulfate; DS, dermatan sulfate; HA, hyaluronic acid; CHS, chondroitin sulfate.
It was shown that heparan sulfate (HS) was the predominant glycosaminoglycan (GAG) present in all investigated tissues, constituting $62-79 \%$ of the total amount of proteoglycans. Dermatan sulphate (DS) accounted for 7-19\% and chondroitin sulfate (CHS) for 5-10\%. Breast and lung also contained a significant amount of hyaluronic acid (HA) (26\% and $13 \%$, accordingly), absent in other tested tissues. A combination of different PG types was specific for every bovine tissue.

Then, an antimitotic effect of the PGs was investigated both on embryonal (mouse fetal tissue) and cancer cells (primary adenocarcinoma, development of transplanted tumors) in vitro and in vivo.

Effect of Bovine Proteoglycans on Cell Proliferation of Mouse Fetal Tissues: From our previous results it was shown that proteoglycans from rat liver and lung are able to inhibit DNA synthesis in fragments of mouse embryo tissues (Zimina and Rykova 1986). Because this effect was not species-specific, bovine liver PGs preparations were tested for their ability to influence on cell proliferation in mice embryonic tissues. Total bovine liver PG preparation was injected to 5-9 days old mice C57B1. Animals were sacrificed after 3 hours by decapitation, tissues were fixed with Carnua reagent, stained with acetocarmin dye and percentage of mitotic cells was calculated (Fig. 2a).

It was shown that bovine liver PG preparation suppressed cell proliferation only in the mouse liver but not in any other tissues. The percentage of dividing cells in liver was decreased from 10.6 $\%$ in control mice to $1.8 \%$ in the experimental ones. With different concentrations of proteoglycans, it was shown that effect has a dosedependent manner with the maximum close to 0,3mg PGs/g mouse body weight (Fig. 2b). This concentration was chosen for experiments in vivo (with the appropriate decrease for cell culture). Suppression of cell proliferation was timedependent with a maximum at 4-5 hours (Fig. 2c).

To test whether PGs from other tissues could suppress cell proliferation, spleen and lung bovine PGs were investigated in similar experiments (Fig. 3). According to our data, antimototic effect of proteoglycans was tissuespecific but species-nonspecific as it has been shown earlier for DNA synthesis (Zimina and Rykova 1986). This property of total proteoglycan preparations may be principal for their anti-tumor 

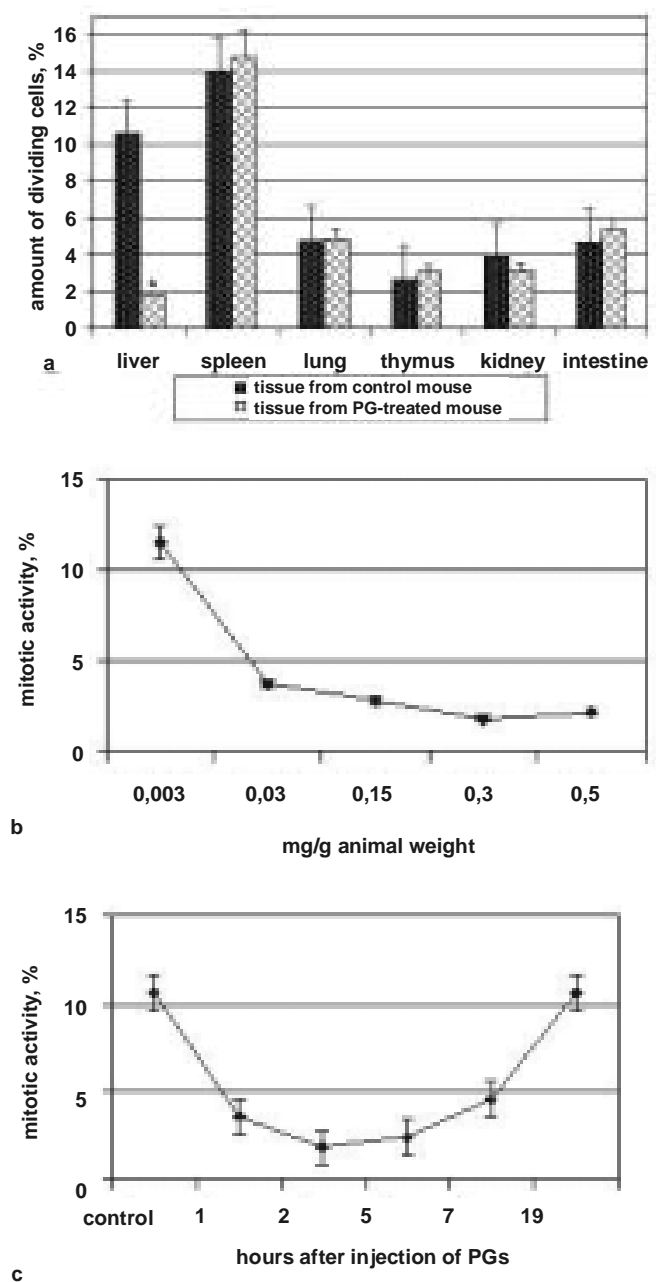

Fig. 2. An influence of bovine liver proteoglycans on mitotic activity of different mouse tissues. a. Total proteoglycan preparation isolated from bovine liver was injected subcutaneously to mice C57Bl (5-9 days old) at a concentration of $0,3 \mathrm{mg} / \mathrm{g}$ mouse weight. Mitotic activity of cells was measured in different organs $3 \mathrm{~h}$ after injection. The results show typical examples of five independent experiments. * $<<0.01$ from the respective control. b. Dose-dependence. c. Time-dependence.

effect and open a new possibility for elaboration of tissue-directed anticancer therapies.

Effect of Bovine Liver Proteoglycan on Tumor Cell Proliferation: To test whether PG preparations have effect on the tumor cells proliferation, experiments with the primary mouse adenocarcinoma cell culture and development of

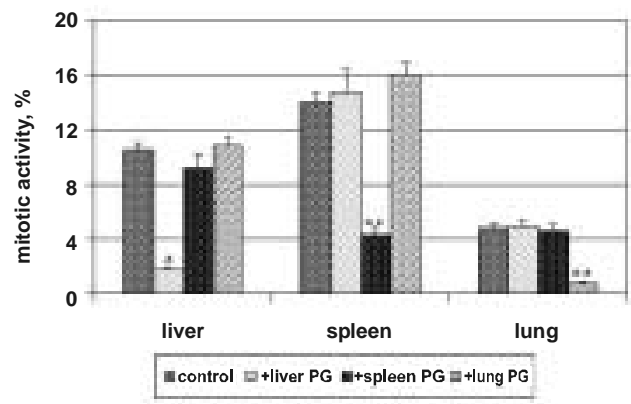

Fig. 3. Tissue-specificity of the effect of different bovine proteoglycan preparations on mouse tissues proliferation. Total proteoglycan preparations isolated from bovine liver/kidney/lung were injected subcutaneously to mice C57Bl (5-9 days old) at a concentration $0,3 \mathrm{mg} / \mathrm{g}$ mouse weight. Mitotic activity of cells was counted in the pieces of different organs $3 \mathrm{~h}$ after injection. $* \mathrm{p}<0.05$ and $* * \mathbf{p}<0.01$ from the respective control.

orthotopic tumor were performed. First, we studied the effects of total bovine liver proteoglycans on primary cell cultures established from spontaneous murine breast adenocarcinoma. Our data demonstrated that PGs inhibited cell proliferation at the minimal concentration of $0.012 \mathrm{mg} / \mathrm{ml}$ and the increase of the dose up to $0,047 \mathrm{mg} / \mathrm{ml}$ had a cytopathogenic effect.

To test an influence of total proteoglycan preparation on tumor cell proliferation in vivo, two different approaches were applied. Primary tumor cells were treated with PG preparation and then the cells were injected to the experimental mice or proteoglycans were injected to the animals with implanted Ehrlich ascite carcinoma.

For the first approach, primary adenocarcinoma cells were isolated from spontaneous breast tumor of $\mathrm{C} 3 \mathrm{H}$ female mouse. The cells were treated either with total PG preparation from bovine liver $(6 \mathrm{mg} / \mathrm{ml}, 10 \mathrm{~min})$ or with saline before injection to the experimental mice (PG-treated or control cells, respectively). A frequency of the tumor formation was calculated for each experimental group of 20 animals (Fig. 4).

Treatment with proteoglycans for $10 \mathrm{~min}$ was already enough to cause a clear difference in the tumor formation rate - at the day 26th only $20 \%$ of mice in the experimental group developed tumors versus $75 \%$ in the control group. At the 43 rd day $55 \%$ of mice developed tumors compared to almost $100 \%$ mice in the control group. It was shown that tumors in the experimental group grew 


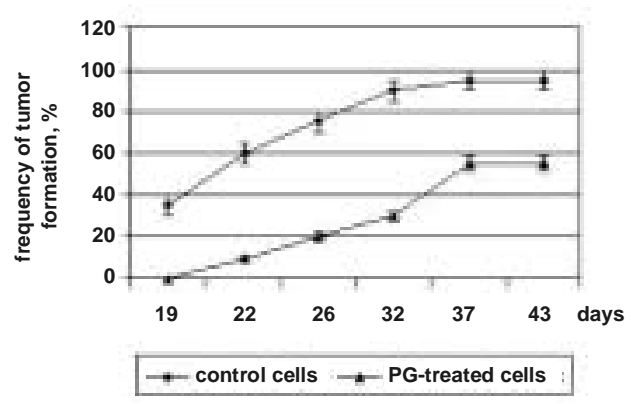

Fig. 4. Frequency of tumor formation in the $\mathrm{C} 3 \mathrm{H}$ mice at different time-points. Brest adenocarcinoma cells were treated either with saline (control cells) or total proteoglycan preparation (PG-treated cells) and inoculated subcutaneously. Percentage of the mice which have been developed tumor was calculated at the different time-points (20 animals/point).

in 7.4 times more slowly then their counterpart in the control mice (1.0 and $7.4 \mathrm{mg} / \mathrm{day}$, accordingly).

To further delineate the influence of total proteoglycan preparation on the tumor development in vivo, it was injected to CC57Br mice with Ehrlich ascite carcinoma at different doses (Fig.5).

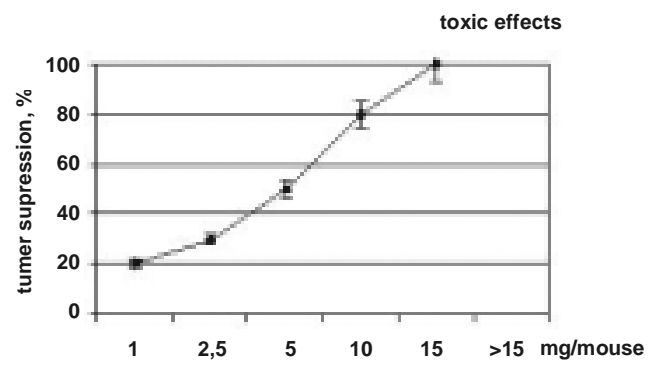

Fig. 5. Tumor suppression of murine carcinoma by the total proteoglycan preparation isolated from bovine liver. PGs were injected intraperitoneally at different doses to Ehrlich ascite carcinoma bearing mice $C C 57 \mathrm{Br}$. The animals were sacrificed after 5 days and ascite volume was determined. The results show summary of five independent experiments.

It was shown that intraperitoneal injection of proteoglycans at doses of $5 \mathrm{mg} / \mathrm{mouse}$ decreased tumor volume by $50 \%$. However, at dose of $15 \mathrm{mg}$ per mouse some toxic effects were appeared resulting in the death of experimental animals. According to this experiment, the optimal concentration of PGs preparation for tumor growth suppression is 5-7 mg/mouse. Taken together, these findings suggest that total PG preparation from bovine adult tissue is able to suppress cell proliferation in both fetal and tumor mouse tissue in vivo. This effect is a tissuespecific and species-non-specific with optimal concentration $0.3-0.4 \mathrm{mg} / \mathrm{g}$ animal weight $(5-7 \mathrm{mg}$ / mouse).

\section{DISCUSSION}

It is known that structural composition of the glycosaminoglycan (GAG)/proteoglycan (PG) component of the extracellular matrix is very important for the normal tissue function and its alteration may have significant consequences on cell proliferation and/or differentiation. Although many studies have evaluated the influence of different PGs/GAGs on the cell proliferation, two main approaches usually were applied. First, a certain proteoglycan molecules were studied such as decorin (Moscatello et al. 1998; Santra et al. 2000), lumican (Vuillermoz et al. 2004; Vij et al. 2004), syndecan (Maeda et al. 2004; Barbareschi et al. 2003), glypican (Filmus 2001), perlecan (Mathiak et al. 1997; Garl et al. 2004; Sharma et al. 1998) and others. In this case, an investigation of their functional activity in vitro and in vivo is facilitated due to the existence of various technical tools (specific primers, antibodies etc). Another approach is to isolate different classes of glycosaminoglycans (HS, DS, CHS, KS, HA) and investigate their influence on cell proliferation in tissue culture (Redini et al. 1990; Volpi et al. 1994; Nikitovic et al. 2005; Nagasawa et al. 1993; Maurer et al. 1994). However due to a high heterogeneity of polysaccharide chains GAG even within the same PG class, very careful attention should be paid to the problem of a sameness of PG/GAG samples.

In this study, we investigated a possible antimitotic activity of total proteoglycan preparations isolated from different bovine tissues. As it was shown in Results, each such preparation consists of a combination of different PG classes in a different ratio. To be confident in a sameness of the preparation 5 independent batches were obtained. All batches of proteoglycan preparations were tested very carefully for their physic-chemical characteristics (amino acid composition, uronic acid content, sialic acids, aminosaccharides and neutral 
saccharides). It was shown that the proteoglycan preparations maintained its functional activity after chlorophorm/methanol (2:1) treatment, boiling for $30 \mathrm{~min}$, autoclaving at $120^{\circ} \mathrm{C}$ and storage for 1 year at room temperature. The stability of the PG preparation and possibility to get a sterile solution suitable for the injection in vivo serves as a base for the investigation of the total proteoglycans effect on cell proliferation. It was shown that total PG preparation from bovine liver composed of mainly heparansulfate (HS) and dermatansulfate (DS). According to literature data, both HS and DS possessed a controversial effect on cell proliferation.

DS significantly stimulated cell growth of monoblastic leukemia cells U937 (Volpi et al. 1994), Tawa sarcoma cells (Nagasawa 1993), malignant mesothelioma cell lines (Syrokou et al. 1999) but inhibited cell proliferation of both normal osteoblasts and transformed osteoblastic cells (Nikitovic et al. 2005). Also, a dependency from the presence of serum was shown - DS was not able to stimulate cell growth of normal HEL cells in serum-free agar system but stimulated the growth of HEL cells and colony formation of tumor HL-60 and U937 cells in serum-containing culture system (Maurer et al. 1994).

In their turn, HS did not change the proliferation of monoblastic leukemia cell line (U937) (Volpi et al. 1994) but accelerated the growth of the cultured Tawa sarcoma cells (Nagasawa 1993). According to other data, effect of HS was highly dependent on culture medium composition and iduronic acid (IdoUA)/ glycuronic acid (GlcUA) content of glycosaminoglycans. In serum-free agar system HS did not change growth of HEL cells but in serumcontaining culture system HS promoted colony formation of HL60 and U937 cells and stimulated the growth of HEL cells (Maurer et al. 1994). (IdoUA)-rich HS had significant antiproliferative effects on mesothelioma cells of both fibroblastlike and epithelial morphology while GlcUA-rich HS had no effect (Syrokou et al. 1999).

In our study, a natural mixture of different proteoglycans from adult bovine liver was used. There were identified mainly IdoUA-containing PGs - heparansulfates and dermatansulfates, which combination caused marked growth suppression both in vitro and in vivo using an adenocarcinoma cell line and an orthotopic mammary carcinoma model. The results suggested that proteoglycans play an important role in regulation of mammary carcinoma cell growth. The possible significance of the suppressor action of total proteoglycans on tumor cell proliferation remains open to further investigation.

\section{ACKNOWLEDGEMENTS}

The authors thank Prof. Eugene Zabarovsky, Karolinska Institute, Stockholm, Sweden, for his valuable comments and helpful discussion regarding this manuscript.

\section{REFERENCES}

Barbareschi M, Maisonneuve P, Aldovini D, Cangi MG, Pecciarini L, Angelo Mauri F, Veronese S, Caffo O, Lucenti A, Palma PD, Galligioni E, Doglioni C 2003. High syndecan-1 expression in breast carcinoma is related to an aggressive phenotype and to poorer prognosis. Cancer, 98(3): 474-483.

Filmus J 2001. Glypicans in growth control and cancer. Glycobiology, 11(3): 19R-23R.

Garl PJ, Wenzlau JM, Walker HA, Whitelock JM, Costell M, Weiser-Evans MCM 2004. Perlecan-Induced Suppression of Smooth Muscle Cell Proliferation Is Mediated Through Increased Activity of the Tumor Suppressor PTEN. Circulation Research, 94: 175.

Iozzo RV 1998. Matrix proteoglycans: from molecular design to cellular function. Annu Rev Biochem, 67: 609-652.

Kirby KS 1956. A new method for the isolation of ribonucleic acids from mammalian tissues. Biochem $J, 64(3)$ : 405-408.

Kresse H, Schonherr E 2001. Proteoglycans of the extracellular matrix and growth control. $J$ Cell Physiol, 189(3): 266-274.

Maeda T, Alexander CM, Friedl A 2004. Induction of syndecan-1 expression in stromal fibroblasts promotes proliferation of human breast cancer cells. Cancer Res, 64(2): 612-21.

Mathiak M, Yenisey C, Grant DS, Sharma B, Iozzo RV 1997. A role for perlecan in the suppression of growth and invasion in fibrosarcoma cells. Cancer Res, 57(11): 2130-2136.

Maurer AM, Han ZC, Dhermy D, Briere J 1994. Glycosaminoglycans enhance human leukemic cell growth in vitro. Leuk Res, 18(11): 837-842.

Moscatello DK, Santra M, Mann DM, McQuillan DJ,Wong AJ, Iozzo RV 1998. Decorin Suppresses Tumor Cell Growth by Activating the Epidermal Growth Factor Receptor. J Clin Invest, 101(2): 406412 .

Nagasawa S 1993. Effect of glycosaminoglycans on the growth of cultured tumor cells. J Osaka Dent Univ, 27(2): 121-133.

Nikitovic D, Zafiropoulos A, Tzanakakis GN, Karamanos NK, Tsatsakis AM 2005. Effects of glycosaminoglycans on cell proliferation of normal osteoblasts and human osteosarcoma cells depend on their type and fine chemical compositions. Anticancer Res, 25(4): 2851-2856. 
Redini F, Moczar E, Poupon MF 1990. Effects of glycosaminoglycans and extracellular matrix components on metastatic rat rhabdomyosarcoma tumor and myoblast cell proliferation. Clin Exp Metastasis, 8(5): 491-502.

Santra M, Eichstetter I, Iozzo RV 2000. An antioncogenic role for decorin. Down-regulation of ErbB2 leads to growth suppression and cytodifferentiation of mammary carcinoma cells. J Biol Chem, 275(45): 35153-35161.

Selleck SB 2000. Proteoglycans and pattern formation: sugar biochemistry meets developmental genetics. Trends Genet, 16(5): 206-212.

Sharma B, Handler M, Eichstetter I, Whitelock JM, Nugent MA, Iozzo RV 1998. Antisense Targeting of Perlecan Blocks Tumor Growth and Angiogenesis In Vivo. J Clin Invest, 102(8): 1599-1608.

Syrokou A, Tzanakakis G, Tsegenidis T, Hjerpe A, Karamanos NK 1999. Effects of glycosaminoglycans on proliferation of epithelial and fibroblast human malignant mesothelioma cells: a structurefunction relationship. Cell Prolif, 32: 85-99.
Timar J, Lapis K, Dudas J, Sebestyen A, Kopper L, Kovalszky I 2002. Proteoglycans and tumor progression: Janus-faced molecules with contradictory functions in cancer. Semin Cancer Biol, 12(3):173-186.

Vij N, Roberts L, Joyce S, Chakravarti S 2004. Lumican suppresses cell proliferation and aids Fas-Fas ligand mediated apoptosis: implications in the cornea. Exp Eye Res, 78(5): 957-971.

Volpi N, Petrini M, Conte A, Valentini P, Venturelli T, Bolognani L, Ronca G 1994. Effects of glycosaminoglycans on U-937 leukemia cell proliferation and differentiation: structure-function relationship. Exp Cell Res, 215(1): 119-130.

Vuillermoz B, Khoruzhenko A, D’Onofrio MF, Ramont L, Venteo L, Perreau C, Antonicelli F, Maquart FX, Wegrowski Y 2004. The small leucine-rich proteoglycan lumican inhibits melanoma progression. Exp Cell Res, 296(2): 294-306.

Zimina NP, Rykova VI 1986. Proteoglycans of animal tissue and their effect on DNA synthesis. Biokhimiia, 51(9): 1555-1561. 